Cardiac involvement and lupus nephritis (LN) were developed in $20 \%$ of patients. About $13.75 \%$ of patients had neurological manifestations, $26.5 \%$ articular complications, $16 \%$ vascular involvement and $10 \%$ of them developed infectious complications. Eight percent of these infections were diagnosed concomitantly with the diagnosis of SLE and $92 \%$ of them after the diagnosis of lupus with an average of 25 months. About $52.9 \%$ of the patients developed more than 2 episodes of infection. The spectrum of infectious complications was: pulmonary in $33.3 \%$, urinary in $22.2 \%$ and cutaneous in $13.9 \%$. Tuberculosis was the most frequent infection $12.5 \%$. Lupus flare complicated the infection in $28.6 \%$ of patients with mean SLEDAI score at 10. Comparative study between group 1 and group 2 revealed that $L N$, corticosteroids and immunosuppressors were associated with a high risk of infection $(\mathrm{p}=0.002, \mathrm{p}=0.017$ and $\mathrm{p}=0.034$ respectively). In multivariate analysis only $\mathrm{LN}$ was an independent predictive factor $(\mathrm{OR}=3.5,95 \% \mathrm{Cl}=1.06-$ 12.87, $\mathrm{p}=0.049$ ).

Conclusions: Infections may complicate the course of SLE with flares presenting in $1 / 3$ of cases. Half of the patients had more than 2 episodes of infection during their follow up. The presence of LN represents a predictive factor of such complication.

Disclosure of Interest: None declared

DOI: 10.1136/annrheumdis-2017-eular.3009

\section{AB0518 NEW 2016 ACR/EULAR CLASSIFICATION CRITERIA FOR SJÖGREN SYNDROME: USEFULNESS AND APPLICABILITY IN CLINICAL PRACTICE}

M. Retuerto Guerrero ${ }^{1}$, L. Sierra Herranz ${ }^{2}$, C. Moriano Morales ${ }^{1}$, C. Iñiguez Ubiaga $^{1}$, M. Garijo Bufort ${ }^{1}$, A. Crespo Golmar ${ }^{1}$, C. Álvarez Castro ${ }^{1}$, E. Díez Álvarez ${ }^{1}$, A. López Robles ${ }^{1}$, M. Martín Martínez ${ }^{1}$, T. Perez Sandoval ${ }^{1}$. ${ }^{1}$ Rheumatology Department; ${ }^{2}$ Pneumology Department, University Health Care Complex of León, Leon, Spain

Background: The Sjögren syndrome (SS) is an autoimmune disease where the cellular and humoral mechanisms affect the exocrine glands. In 2016, new classification criteria validated by ACR and EULAR were established.

Objectives: To compare the new criteria with those used so far in our hospital, as well as to assess the need for changes in the current diagnostic strategy. Methods: Retrospective observational study in which 65 patients diagnosed with SS at the Hospital of León were randomly included. We reviewed the diagnostic tests performed and the fulfilment of the different classification criteria developed since 1993. Other variables studied were: sex; age at the time of diagnosis and the months from the onset of symptoms; xerostomia and xerophthalmia; extraglandular involvement, ESSDAI; immunosuppression; Raynaud; lymphoma development; and analytical alterations.

Results: The mean age at the time of diagnosis was 54.9 years $\pm 14|23-82|$, with an average of months from the onset of symptoms to the diagnosis of $10.2 \pm 9.5$ | 0-36|. 90.8\% were women. $87.7 \%$ presented xerostomia; and $91 \%$ showed xerophthalmia, being severe in $43.1 \% .64 .6 \%$ had extraglandular manifestations; being the most prevalent the joint manifestation $(60 \%)$ and the cutaneous one $(18.4 \%)$. Over the past year, $37 \%$ developed haematological alterations in the form of cytopenias, and $73 \%$ biological alterations. At the time of the study, $32.8 \%$ presented low activity, $38.5 \%$ moderate activity and $9.2 \%$ high activity, measured by ESSDAl; being higher in anti-Ro positive patients $(p=0.011)$. There was no association between ESSDAl and other antibodies, Raynaud or severe ocular involvement. 10.8\% required systemic immunosuppression (RTX 5, AZA 2) and $18.5 \%$ needed ocular immunosuppression (topical cyclosporine). Only one patient developed lymphoma.

A Schirmer's test (ST) was performed in $92.3 \%$ (positive in $89.2 \%$ ), saving the Van Bijsterveld test for patients with severe ocular involvement. The Ocular Staining Score (OSS) was not performed in any patient.

The scintigraphy of the salivary glands was positive in $70.8 \%$ of the patients and was not performed in $21.5 \%$. The parotid sialography was only performed in two patients and the study of the salivary flow was not stimulated in none of them.

Regarding the autoimmunity, $80 \%$ presented positive antiRo; $61.5 \%$ antiLa; $89 \%$ ANA: $61.5 \%$ RF; $43 \%$ quadruple positivity.

Labial gland biopsy was performed only in $18.4 \%$, with a positive result in $75 \%$. All patients met the 1993 European Criteria; $86.2 \%$ met the European-American criteria of 2002; and only $10.8 \%$ met the SICCA-ACR Criteria. The new criteria validated by ACR and EULAR were verified in $80 \%$. Four patients who fulfilled the European criteria did not meet the new criteria, coinciding with those patients with negative ST, but positive scintigraphy.

Conclusions: In our hospital, the method for electing the xerostomia study was the salivary scintigraphy; therefore, we cannot establish direct comparisons with the new criteria.

The incorporation of non-stimulated salivary flow in our diagnostic strategy is necessary.

We should consider conducting a lip biopsy more systematically for histological confirmation since there are no validated diagnostic criteria.

Disclosure of Interest: None declared

DOI: 10.1136/annrheumdis-2017-eular.5255

\section{AB0519 LABORATORY ABNORMALITIES IN PATIENTS WITH PRIMARY SJOGREN'S SYNDROME}

M. Fernandez Castro ${ }^{1}$, J.L. Andreu ${ }^{2}$, C. Sanchez-Piedra ${ }^{3}$, V. Martínez Taboada $^{4}$, A. Olive $^{5}$, J. Rosas ${ }^{6}$ on behalf of SJOGRENSER group, part of the Spanish Society of Rheumatology Systemic Autoimmune Diseases Study Group (EASSER). ${ }^{1}$ Rheumatology, Hospital Infanta Sofía; ${ }^{2}$ Rheumatology, Hospital Puerta de Hierro Majadahonda; ${ }^{3}$ Research unit, Spanish Society of Rheumatology; ${ }^{4}$ Rheumatology, Hospital Marqués de Valdecilla, Madrid; ${ }^{5}$ Rheumatology, Hospital Hospital Germans Trias i Pujol, Barcelona; ${ }^{6}$ Rheumatology, Hospital Hospital Marina Baixa, Alicante, Spain

Background: Primary Sjögren's syndrome (pSS) is a chronic systemic autoimmune disease often accompanied by analytical abnormalities. Altered levels in serum protein concentration, blood cell count and autoantibodies contribute to the broad spectrum of biological manifestations that characterize pSS.

Objectives: The objective of this study is to evaluate the presence of laboratory abnormalities in patients with pSS from the SjogrenSER registry.

Methods: We conducted a multicentre transversal study of a cohort of pSS patients fulfilling 2002 European/American criteria, from 33 Spanish rheumatology departments. Every patient was interviewed for data collection and signed an informed consent. Data were also collected by reviewing medical records. Local ethics committees approved the study. Variables were analysed by descriptive statistical methods, using means, medians and rates. Chi-square was used to establish the statistical associations. A $p<0.05$ was considered significant.

Results: Four hundred and thirty-seven patients were included. Ninety-five percent of them were women. The median age of the cohort was 58 years. AntiRo antibodies were present in $93.6 \%$ of patients and AntiLa antibodies in $67.3 \%$ of patients. All patients were ANA+. Rheumatoid factor (RF) was positive in $64.8 \%$ of patients. Low levels of C3 and C4 were observed in $14.87 \%$ and $14.19 \%$ of patients, respectively. Polyclonal hypergammaglobulinemia (HGG) was present in $53 \%$ of patients. Thirteen patients had cryoglobulins $(2.97 \%)$. An increase in $\beta 2$ microglobulin was observed in $22.2 \%$ of patients. Fifty-six percent of the patients had hematological involvement: $29 \%$ of the patients had anemia, $38 \%$ had leukopenia (38\% lymphopenia, $10.5 \%$ had neutropenia), and $9 \%$ had thrombocytopenia. The median ESR was $25 \mathrm{~mm}$. Age at diagnosis and age at onset of symptoms were significantly lower in patients presenting RF+ vs RF- (48.71 vs $53.73, p<0.001$ and 44.76 vs $49.53, p=0.001$, respectively), decreased $\mathrm{C} 3$ vs normal $\mathrm{C} 3$ (45.66 vs $51.18, \mathrm{p}=0.004$ and 42.2 vs 46.99 , $\mathrm{p}=0.018$, respectively), decreased C4 vs normal $\mathrm{C} 4$ (47.02 vs. $50.89, \mathrm{P}=0.042$, for age at diagnosis) and HGG (47.59 vs $54, p<0.001$, and 43.44 vs 50.16 , $\mathrm{p}<0.001$, respectively). ESR was significantly higher in patients with hematological involvement (35.94 vs $26.24, p<0.001$ ), $R F+(36.39$ vs $22.91, p<0.001$ ), decreased C3 (37.8 vs $30.53, p=0.026)$ and C4 (38.71 vs. $30.42, p=0.04)$ ), HGG $(36.21$ vs $26.03, \mathrm{p}<0.001)$ and increased $\beta 2$ microglobulin $(38.80$ vs $29.71, \mathrm{p}=0.009)$. ESSDAI (Eular Sjögren Syndrome Disease Activity Index) was significantly higher in patients with haematological involvement (5.58 vs $3.69, p<0.001), R F+(5.40$ vs $3.53, p<0.001)$ and $H G G(5.31$ vs $3.93, P=0.011)$. The median ESSDAl score was 2 (P25-P75, 0-4).

Conclusions: in SjogrenSER registry all patients were serologically positive. More than half of the patients presented abnormalities in serum proteins and $14 \%$ had hipocomplementemia. More than half of the patients had abnormalities, mostly leucopenia and lymphopenia. Patients with analytical alterations were younger at the time of diagnosis and had more often elevated ESR and higher ESSDAl score.

Disclosure of Interest: None declared

DOI: 10.1136/annrheumdis-2017-eular.2603

\section{AB0520 THE ROLE OF LEPTIN IN SJOGREN'S DISEASE}

M. Erdoğan ${ }^{1}$, M.E. Tezcan ${ }^{2}$, K. Başaka ${ }^{3} .{ }^{1}$ Internal medicine division of Rheumatology, Cerrahpaşa Medical Faculty; ${ }^{2}$ Internal medicine division of Rheumatology; ${ }^{3}$ Pathology, Dr. Lürfi Kırdar Kartal Training and Research Hospital, Istanbul, Turkey

Background: Sjogren's disease (SD) is a multisystemic disease mostly manifest with sicca symptoms. Lympocytic infiltration of the glandular and extra glanduler organs is the dominant pathologic feature of the disease. Multiple stimulators were accused in the pathogenesis of SD. Leptin, an endogenous peptide, involves in various metabolic processes as well as influence immune system (1). Increased serum leptin level is observed in patients with autoimmune diseases such as SD, systemic lupus erythematosus and rheumatoid arthritis when compared to healthy controls (2).

Objectives: Even if serum leptin level increases in the patient with SD, there is no data about its effect on exocrine glands. We aimed to compare density of leptin in the salivary gland of SD patients with control group. Furthermore we evaluted the relation between intensity of lympocytic infiltration and density of leptin in the salivary glands of SD patients

Methods: We applied leptin immunostain to minor salivary glands samples of 24 SD patientss, who were fullfilled American College of Rheumatology Sjögren's Disease Classification Criteria (ACR-SDCC) and 19 patients who undergo minor salivary gland biopsy due to clinically on suspicion of SD but not fullfilling the ACRSDCC and had no lymphocytic focus on biopsy. Herein,leptin density in acinar and ductal structures of the salivary glands were evaluated in both groups.Moreover, 